Check for updates

Cite this: RSC Adv., 2019, 9, 16525

Received 29th April 2019

Accepted 12th May 2019

DOI: $10.1039 / c 9 r a 03214 b$

rsc.li/rsc-advances

\section{Catalyst-free four-component domino synthetic approach toward versatile multicyclic spirooxindole pyran scaffolds $\uparrow$}

\begin{abstract}
Aref Mohammadi, Mohammad Bayat (iD * and Shima Nasri (iD
A new versatile strategy involving a sequential four-component reaction of the nitroketene dithioacetals, alkylamine/benzylamine, isatin and various enolizable active methylene structures (pyrazolone, barbituric acid, 1,3-indandione and 2-hydroxy-1,4-naphthoquinone) as precursors under mild and catalyst-free conditions results in the synthesis of new functionalized spirooxindole pyrans named spirolindoline-3, $4^{\prime}$ pyrano[2,3-c]pyrazol], spiro[indoline-3,5'-pyrano[2,3-d]pyrimidine], spiro[indeno[1,2-b]pyran-4,3'indoline] and spiro[benzo[g]chromene-4, $3^{\prime}$-indoline] in moderate to good yields. The use of various active methylene compounds affords a range of skeletally distinct spirooxindole-based heterocycles with potential biological properties. The present strategy has many advantages, such as convenient one-pot operation, simple workup procedures and straightforward isolation without using tedious purification steps such as column chromatography, progress under catalyst-free condition and high molecular diversity.
\end{abstract}

\section{Introduction}

A significant challenge to explore biological targets is the design of an economical and highly efficient chemical reaction to supply structurally diverse compounds with excellent biological properties. ${ }^{1}$ Therefore, to satisfy the need for small molecules containing bioactive heterocycles as biological targets, access to a wide variety of compounds with potential biological activities is highly desirable. ${ }^{2,3}$ The $4 H$-pyran derivatives are defined as useful biological and pharmacological targets with biological properties such as antiviral, ${ }^{4}$ antibacterial, ${ }^{5}$ antitumor, ${ }^{6}$ and diuretic activities. ${ }^{7}$ In addition, isatin and its derivatives have attractive biological properties and are widely used in the synthesis of spirooxindole-based organic compounds. ${ }^{8}$ Spirooxindole system is one of the most phenomenal spirocycles and the central structural framework found in a diversity of natural products and bioactive molecules. ${ }^{9}$ The existence of a chiral spiro center around a biologically active scaffold leads to structural rigidity and complexity, which is an ongoing challenge for synthetic organic chemists. ${ }^{10}$ Hence, heterocyclic spirooxindole pyrans are attractive synthetic targets for synthetic chemists due to their biological activities such as antiviral, antibacterial, antifungal, anti-HIV, and anti-cancer activities. ${ }^{11}$ Various groups have developed synthetic approaches to access this scaffold., ${ }^{3,11}$ In spite of the

Department of Chemistry, Faculty of Science, Imam Khomeini International University, Qazvin, Iran. E-mail: m.bayat@sci.ikiu.ac.ir; bayat_mo@yahoo.com; Tel: +98 28 33780040

† Electronic supplementary information (ESI) available. See DOI: 10.1039/c9ra03214b development of versatile reactions to synthesize these structures, new, simple and highly efficient methods are still required.

In organic synthesis, development of synthetically useful and unique organic reaction methods in eco-friendly media that generate complex molecular libraries with a minimum number of synthetic steps is a major challenge that can be resolved by multicomponent reactions (MCRs) as the most efficient strategy in modern organic synthesis. ${ }^{12}$ The inherent advantages of MCRs including operational simplicity, step efficiency, lower energy consumption and costs, and high atom economy without waste production have resulted in designing novel MCRs using eco-friendly solvents as one of the goals of sustainable chemistry and synthetic chemistry. ${ }^{13}$

There are many reports on multicomponent entries in the synthesis of spirooxindole- $4 \mathrm{H}$-pyrans from readily available starting materials including isatin, malononitrile, and 1,3dicarbonyl compounds. This class of reaction has been carried out under different conditions as follows: in the presence of $p$ -

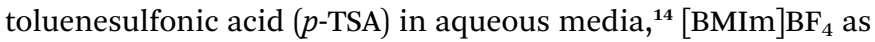
an ionic liquid catalyst at ambient temperature, ${ }^{15}$ piperidine under ultrasound irradiation, ${ }^{16}$ proline-derived thiourea catalyst, ${ }^{3} \mathrm{CuFe}_{2} \mathrm{O}_{4}(10 \mathrm{~mol} \%)$ as an inexpensive, magnetically recoverable, and reusable catalyst in refluxing water, ${ }^{17} \mathrm{SBA}-\mathrm{Pr}-$ $\mathrm{NH}_{2}$ as an efficient heterogeneous nanoporous solid basic catalyst in an aqueous medium, ${ }^{18} \alpha$-amylase extracted from hog pancreas, ${ }^{19}$ silica-bonded $N$-propyltriethylenetetramine as a heterogeneous solid base catalyst, ${ }^{20}$ triethylamine in ethanol, ${ }^{21}$ silica-bonded ionic liquids as a recyclable catalyst, ${ }^{22}$ $1 \mathrm{~mol} \%$ of (DHQD) ${ }_{2} \mathrm{PYR},{ }^{23}$ ytterbium triflate as a catalyst at room temperature, ${ }^{24}$ under visible-light irradiation in water- 


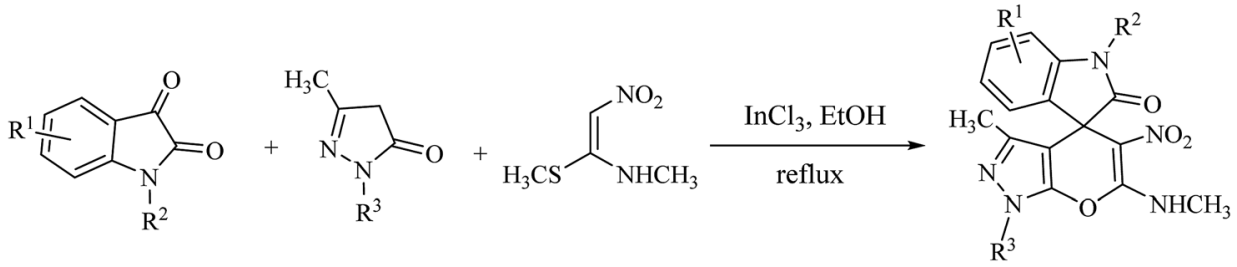

Scheme 1 Synthesis of spiro(indoline-3,4'-pyrano[2,3-c]pyrazol)-2-one derivatives catalyzed by indium trichloride.

ethyl lactate at room temperature, ${ }^{25}$ in the presence of a catalytic amount of 1,4-diazabicyclo[2.2.2] octane (DABCO) in ethanol or $\mathrm{H}_{2} \mathrm{O} / \mathrm{EtOH}$ mixture under reflux conditions, ${ }^{26}$ in dimethylsulfoxide (DMSO) as a highly polar aprotic solvent, ${ }^{11}$ by dabco-based ionic liquids, ${ }^{27}$ using guanidine-functionalized magnetic $\mathrm{Fe}_{3} \mathrm{O}_{4}$ nanoparticles (MNPs) as an efficient heterogeneous catalyst. ${ }^{28}$

Although various catalysts have been applied for the synthesis of these compounds, the role of catalysts is the same in these reactions and it was to catalyze the Knoevenagel condensation, Michael addition, and cyclization reactions. Each of the procedures has its own benefits with at least one drawback, such as low yield, long reaction time, harsh reaction conditions, and use of toxic and expensive catalysts or solvents; hence, there is still a requirement for simple, efficient and economical methods.

In recent years, ketene aminals (KAs) have been used as powerful and versatile enamine analogues with unique structural properties in the synthesis of various types of fused fiveand six-membered heterocycles and drug-like compounds. ${ }^{29}$ In this way, specific strategies have been developed for the synthesis of substituted spirooxindole- $4 \mathrm{H}$-pyrans based on utilizing KAs as starting materials as follows. In 2014, Poomathi et al. reported a versatile high-yielding indium trichloridemediated one-pot regioselective reaction for the synthesis of spiroxindoles via domino one-pot, three-component reaction of isatins, pyrazoles, and (E-)-N-methyl-1-(methylthio)-2nitroethenamine (Scheme 1). ${ }^{30}$

In 2017, Safari et al. reported the synthesis of novel symmetrical bis-spirooxindole derivatives from the reaction of isatins, dihalides, malono derivatives and $\mathrm{C}-\mathrm{H}$ activated carbonyl compounds or ketene aminal derivatives in the presence of potassium carbonate $\left(\mathrm{K}_{2} \mathrm{CO}_{3}\right)$ in polyethylene glycol 400 (PEG-400) at room temperature (Scheme 2). ${ }^{31}$

With this article background and owing to our interest in catalyst-free approaches by using environmentally friendly solvents as a green and safe method for the formation of molecular architectures with a wide range of potential biological activities, ${ }^{32-35}$ herein, we designed and introduced an improved, catalyst-free, and easy method to access the spirooxindoles and $4 H$-pyrans, for which the results are shown in the Results and discussion section.

\section{Results and discussion}

Due to the pharmacological activities of spirooxindole motifs and the bioactivity of pharmacophores such as naphthoquinone, indandion, pyrazolone, and pyrimidine derivatives, we synthesized products 5 which comprise both of spirooxindole and one of these pharmacophores. The hybrid molecules may inherit biological properties of both spirooxindole and pharmacophore structures. Synthesis of $\mathbf{5}$ could be easily achieved by tandem Knoevenagel condensation/Michael addition/imine-enamine tautomerism/O-cyclization of the reaction of amines 1, 1,1-bis(methylthio)-2-nitroethene 2, isatin $\mathbf{3}$ and enolizable active methylene structures $\mathbf{4 a}-\mathbf{f}$ (pyrazolone, barbituric acid, 1,3-indandione and 2-hydroxy-1,4naphthoquinone) (Scheme 3).

In this paper, KAs were derived from the addition of various alkylamine/benzylamine 1 to 1,1-bis(methylthio)-2-nitroethene 2 , wherein the electron density of the $\alpha$-carbon (C1) increased

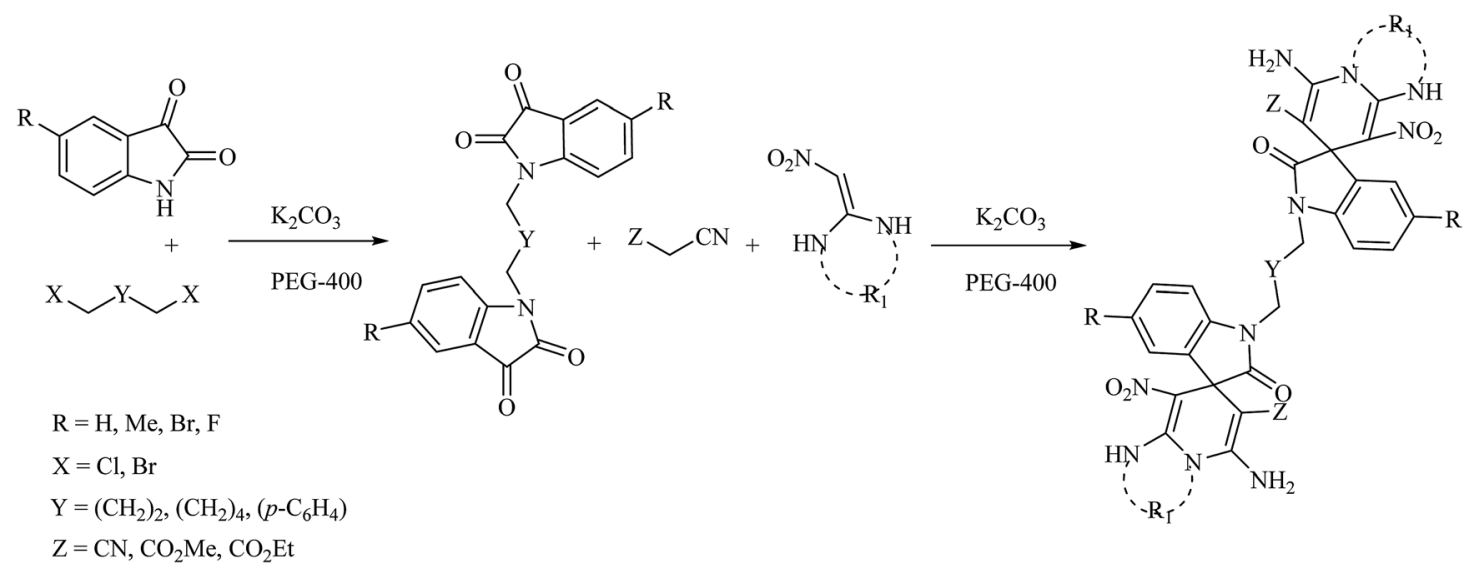

Scheme 2 The one-pot, multi-component synthesis of bis-spirooxindoles in PEG-400/ $\mathrm{K}_{2} \mathrm{CO}_{3}$ at room temperature. 
<smiles>[R1]N[N+](=O)C=C([SiH3])[SiH3]</smiles>

1<smiles>[R]c1ccc2c(c1)C(=O)C(=O)N2</smiles>

3

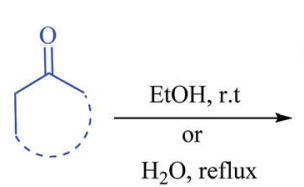

4a-f<smiles>[R]c1ccc2c(c1)C1(C(=O)N2)C2=C(CCCC2)OC(NC)=C1[N+](=O)[O-]</smiles>

5a-q

$\mathrm{R}^{1}=\mathrm{CH}_{3}, \mathrm{CH}_{2} \mathrm{CH}_{2} \mathrm{CH}_{3}, \mathrm{CH}\left(\mathrm{CH}_{3}\right)_{2}, \mathrm{CH}_{2} \mathrm{Ph}$

$\mathrm{R}^{2}=\mathrm{H}, \mathrm{Cl}, \mathrm{Br}$<smiles>CC1=NN(c2ccccc2)C(=O)C1</smiles>

$4 a$<smiles>CC1=NN(c2cccc(Cl)c2)C(=O)C1</smiles>

$4 \mathrm{~b}$<smiles>CN1N=C(C(F)(F)F)CC1=O</smiles>

$4 c$<smiles>O=C1CC(=O)NC(=O)N1</smiles>

4d<smiles>O=C1CC(=O)c2ccccc21</smiles>

$4 e$<smiles>O=C1C=C(O)C(=O)c2ccccc21</smiles>

$4 \mathrm{f}$

Scheme 3 Synthetic approaches for the formation of spiro[indoline-3,4'-pyrano[2,3-c]pyrazol], spiro[indoline-3,5'-pyrano[2,3-d]pyrimidine], spiro[indeno[1,2-b]pyran-4,3'-indoline] and spiro[benzo[g]chromene-4,3'-indoline] (5a-q).

due to the conjugation effect of both the electron-donating nitrogen and sulfur atoms. ${ }^{29}$ Isatin, as an important distinguished building block, is a type of unsymmetrical cyclic ketone with excellent reactivity that has been used in the synthesis of different types of spirooxaindole skeletons. ${ }^{36}$ In this study, a variety of multicyclic spirooxindole pyran scaffolds (5a-q) were synthesized; these derivatives provide a class of structurally diverse compounds that demonstrate promise for future bioassays and medical therapy applications.
The acceptable reaction mechanism is designated in Scheme 4. In the case of the synthesis of 5 , it is possible that initially, the formation of ketene aminal (KA) 6 occured through the addition of amine 1 to 1,1-bis (methylthio)-2-nitroethene 2. Then, Michael acceptor 7 formed through Knoevenagel condensation between isatin 3 and enolizable active methylene structures $\mathbf{4}$, which was followed by the loss of water molecules. Then the KA 6 as an enamine added to the Knoevenagel adduct 7 in a Michael addition type reaction to give open chain intermediate $\mathbf{8}$, which after successive imine-enamine tautomerization

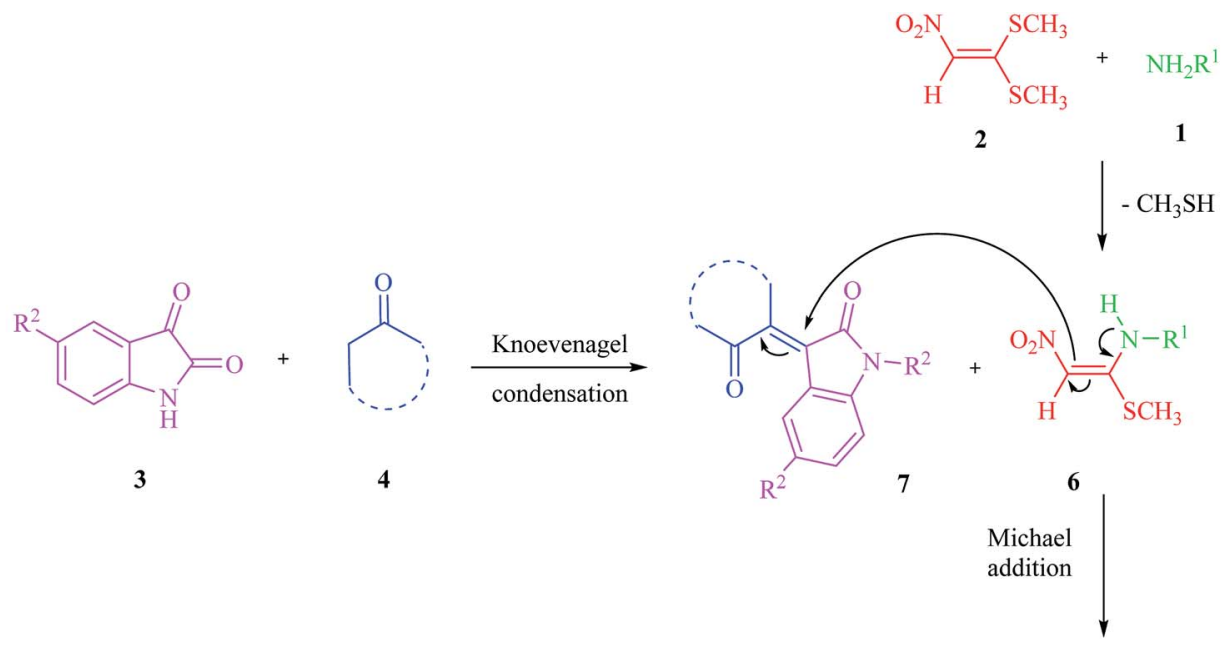

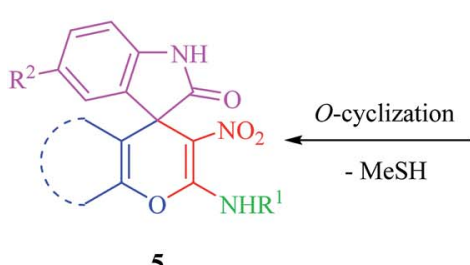

5

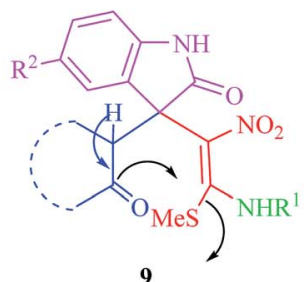

9

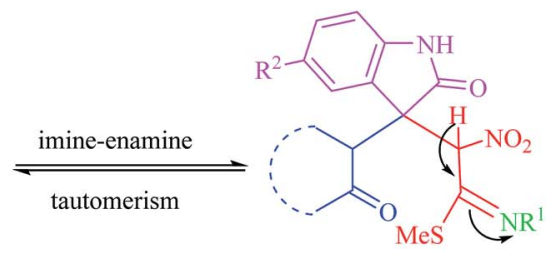

8

Scheme 4 A plausible mechanism for the formation of 5 in catalyst-free conditions. 
Table 1 Substrate scope study of multicyclic spirooxindole pyran scaffolds with a series of amines, isatin derivatives, and active methylene structures

Entry

Active methylene structures

$\mathrm{R}^{1}$

Product

$\operatorname{Time}^{a}(\mathrm{~h})$

Yield (\%)<smiles>CC1=NN(c2ccccc2)C(=O)C1</smiles>

$\mathrm{CH}_{3}$<smiles>CNC1=C([N+](=O)[O-])C2(C(=O)Nc3ccccc32)c2c(C)nn(-c3ccccc3)c2O1</smiles>

$\mathrm{Cl}$<smiles>CC1=NN(c2ccccc2)C(=O)C1</smiles>

$\mathrm{CH}_{3}$<smiles>CNC1=C([N+](=O)[O-])C2(C(=O)Nc3ccc(Cl)cc32)c2c(C)nn(-c3ccccc3)c2O1</smiles>

$\mathrm{CH}_{3}$<smiles>CC1=NN(c2ccccc2)C(=O)C1</smiles>

$\mathrm{H}_{3} \mathrm{C}$<smiles>CC1=NN(c2cccc(Cl)c2)C(=O)C1</smiles><smiles>CC1=NN(c2cccc(Cl)c2)C(=O)C1</smiles>

$\mathrm{CH}_{3}$

$\mathrm{H}$<smiles>CNC1=C([N+](=O)[O-])C2(C(=O)Nc3ccc(Br)cc32)c2c(C)nn(-c3ccccc3)c2O1</smiles>

9

8

$\mathrm{Br}$<smiles>CNC1=C([N+](=O)[O-])C2(C(=O)Nc3ccccc32)c2c(C)nn(-c3cccc(Cl)c3)c2O1</smiles>

$\mathrm{CH}_{3}$

$\mathrm{Br}$<smiles>CNC1=C([N+](=O)[O-])C2(C(=O)Nc3ccc(Br)cc32)c2c(C)nn(-c3cccc(Cl)c3)c2O1</smiles>

$\mathrm{Cl}$<smiles>CNC1=C([N+](=O)[O-])C2(C(=O)Nc3ccc(Cl)cc32)c2c(C)nn(-c3cccc(Cl)c3)c2O1</smiles>

80

85

78

86

75

80 
Table 1 (Contd.)

Entry Active methylene structure

$\mathrm{R}^{1}$<smiles>CN1N=C(C(F)(F)F)CC1=O</smiles><smiles>CC1=NN(c2ccccc2)C(=O)C1</smiles><smiles>O=C1CC(=O)NC(=O)N1</smiles>

$\mathrm{CH}_{3}$

$\mathrm{CH}_{3}$

$\mathrm{Br}$

$\mathrm{R}^{2}$

Product

Time $^{a}(\mathrm{~h})$

Yield (\%)

8

$\mathrm{CH}_{2} \mathrm{Ph}$

$\mathrm{Cl}$<smiles>CNC1=C([N+](=O)[O-])C2(C(=O)Nc3ccc(Br)cc32)c2c(C(F)(F)F)nn(C)c2O1</smiles>

10

62

12

58<smiles></smiles>

10

65

$\mathrm{CH}_{2} \mathrm{CH}_{2} \mathrm{CH}_{3}$

$\mathrm{Cl}$<smiles>CNC1=C([N+](=O)[O-])C2(C(=O)Nc3ccc(Cl)cc32)c2c([nH]c(=O)[nH]c2=O)O1</smiles>

10

75

$\mathrm{CH}\left(\mathrm{CH}_{3}\right)_{2}$

$\mathrm{Cl}$<smiles>CCCNC1=C([N+](=O)[O-])C2(C(=O)Nc3ccc(Cl)cc32)c2c([nH]c(=O)[nH]c2=O)O1</smiles>

5j<smiles>O=C1CC(=O)NC(=O)N1</smiles>

$\mathrm{CH}_{2} \mathrm{CH}_{2} \mathrm{CH}_{3}$

$\mathrm{Br}$<smiles>CC(C)NC1=C([N+](=O)[O-])C2(C(=O)Nc3ccc(Cl)cc32)c2c([nH]c(=O)[nH]c2=O)O1</smiles>

10

76<smiles>O=C1CC(=O)NC(=O)N1</smiles>

$\mathrm{CH}\left(\mathrm{CH}_{3}\right)_{2}$<smiles>O=C1CC(=O)NC(=O)N1</smiles>

$\mathrm{Br}$<smiles>CCCNC1=C([N+](=O)[O-])C2(C(=O)Nc3ccc(Br)cc32)c2c([nH]c(=O)[nH]c2=O)O1</smiles>

12

71

51<smiles>CC(C)NC1=C([N+](=O)[O-])C2(C(=O)Nc3ccc(Br)cc32)c2c([nH]c(=O)[nH]c2=O)O1</smiles>

12

72 
Table 1 (Contd.)

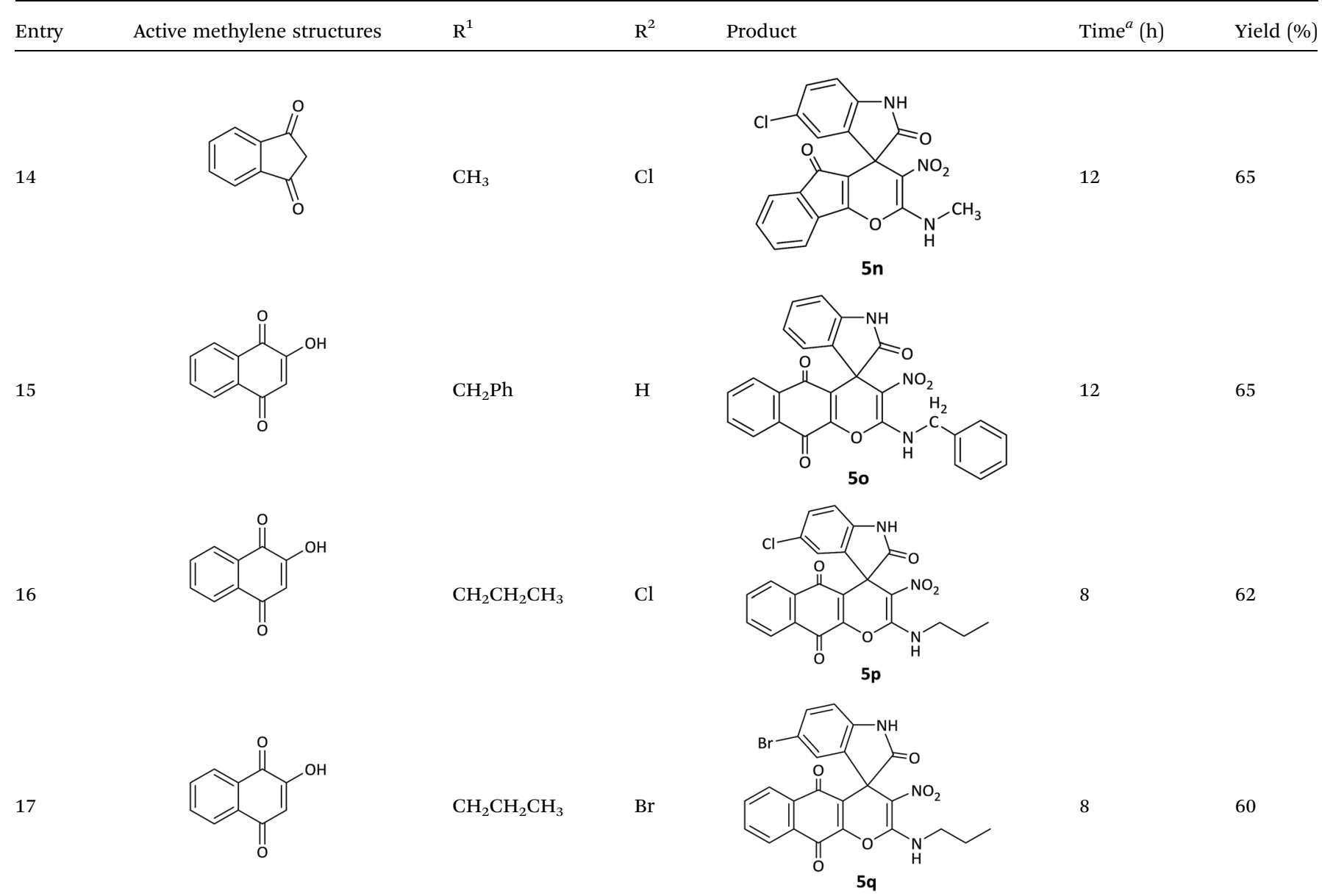

${ }^{a}$ The reaction time of the second step.

underwent $O$-cyclization via an attack of the enol form of $\mathrm{C}-\mathrm{H}$ activated compound on KA, leading to product 5 (Scheme 4).

We surveyed the scope of these reactions by varying the derivatives of amine $\mathbf{1}$, isatin $\mathbf{3}$, and the active methylene compounds 4 to produce product 5 (Table 1). The reaction proceeds cleanly and completely in the presence of different reagents to afford a series of spiro products $5 \mathbf{a}-\mathbf{q}$ in $58-86 \%$ yields. It is noteworthy that the expected product $\mathbf{5}$ was obtained in good yield when pyrazolone was used, but using other $\mathrm{C}-\mathrm{H}$ activated compounds, the expected product 5 was acquired in moderate yields. The substituents on the aromatic ring of the isatin like chlorine or bromine affected the reaction and gave desired products in a long time with fewer yields. The products 5d-5q are novel compounds (Table 1) that have not been reported in the previous literature.

The structures of synthesized derivatives were concluded by elemental and spectral analysis such as FT-IR, ${ }^{1} \mathrm{H},{ }^{13} \mathrm{C}$ NMR and mass spectroscopy. The mass spectrum of compound 5a exhibited a molecular ion peak at $m / z 403$, which was in accordance with the offered structure. The ${ }^{1} \mathrm{H}$ NMR spectrum of 5a displayed one singlet for $\mathrm{CH}_{3}$ group $(\delta 1.58 \mathrm{ppm})$, one doublet for $3 \mathrm{H}$ of $\mathrm{CH}_{3}$ group attached to $\mathrm{NH}\left(\delta 3.17 \mathrm{ppm},{ }^{3} J_{\mathrm{HH}}=\right.$ $4.8 \mathrm{~Hz}$ ), aromatic region of the spectrum for the aromatic moieties $(\delta 6.87-7.73 \mathrm{ppm})$, one multiplet for $\mathrm{NH}$ attached to $\mathrm{CH}_{3}(\delta 10.71-10.73 \mathrm{ppm})$ and one singlet for $\mathrm{NH}$ group of isatin ( $\delta$ 10.74 ppm). The ${ }^{1} \mathrm{H}$-decoupled ${ }^{13} \mathrm{C}$ NMR spectrum of $\mathbf{5 a}$ exhibited 21 distinguished resonances. One peak appeared at $\delta 176.6 \mathrm{ppm}$, which was attributed to one amide carbonyl group and the specific peaks of $C_{\text {spiro }}, \mathrm{NHCH}_{3}$ and $\mathrm{CH}_{3}$, were assigned at $\delta 50.0,29.4$ and $12.2 \mathrm{ppm}$, which confirmed the selective synthesis of $5 \mathbf{a}$.

\section{Conclusions}

In General, we developed an environment friendly process for the synthesis of a library of spiro[indoline-3,4'-pyrano[2,3-c] pyrazol], spiro[indoline-3,5'-pyrano[2,3- $d$ ]pyrimidine], spiro [indeno[1,2-b]pyran-4,3'-indoline $]$ and spiro[benzo $[g]$ chromene$4,3^{\prime}$-indoline] compounds in moderate to good yields (58-86\%). This reaction proceeded via sequential four-component reaction between alkylamine/benzylamine, 1,1-bis(methylthio)-2nitro ethylene, isatin and various active methylene 
compounds in $\mathrm{EtOH}$ or $\mathrm{H}_{2} \mathrm{O}$ medium. Prominent advantages of this method are the diversity of molecular structure, an easy workup, the absence of a catalyst and operational simplicity.

\section{Experimental section}

\section{General}

The nitroketene dithioacetals, isatin derivatives, various amines, various active methylene compounds and solvents were obtained from Sigma Aldrich and Fluka Co., which were used without further purification. IR spectra: Bruker Tensor 27 spectrometer. NMR spectra: Bruker DRX-300 Avance instrument (300 $\mathrm{MHz}$ for ${ }^{1} \mathrm{H}$ and $75.4 \mathrm{MHz}$ for ${ }^{13} \mathrm{C}$ ) with DMSO- $d_{6}$ as solvents. Chemical shifts are expressed in parts per million (ppm), and coupling constant $(J)$ are reported in hertz $(\mathrm{Hz})$. Mass spectra: Agilent 5975C VL MSD with Triple-Axis detector operating at an ionization potential of $70 \mathrm{eV}$. Elemental analyses for $\mathrm{C}, \mathrm{H}$ and $\mathrm{N}$ : Heraeus CHNO-Rapid analyzer. Melting points: electrothermal 9100 apparatus.

\section{General procedure for the synthesis of $5 a-h$ and $5 n-q$}

A mixture of amine (1 mmol), 1,1-bis(methylthio)-2-nitro ethylene $(0.165 \mathrm{~g}, 1 \mathrm{mmol})$, and $10 \mathrm{~mL} \mathrm{EtOH}$ was taken in a $50 \mathrm{~mL}$ round-bottomed flask, and was stirred for $4 \mathrm{~h}$. Then, isatin derivatives $(1 \mathrm{mmol})$ and active methylene compounds (1 $\mathrm{mmol}$ ) were added to the reaction mixture, and was stirred for a period of time shown in Table 1 , which was monitored by TLC (ethyl acetate/n-hexane, $6: 4$ ). The reaction mixture was cooled to room temperature and the precipitate was filtered to obtain the crude product. The solid was washed with $96 \%$ ethanol and dried in an oven at $150{ }^{\circ} \mathrm{C}$ to yield product 5 and analyzed by ${ }^{1} \mathrm{H}$ NMR and ${ }^{13} \mathrm{C}$ NMR spectroscopy. Only in the case of $\mathbf{5 n}$, the round-bottom flask was fitted with a reflux condenser and heated with stirring in an oil-bath at reflux temperature.

\section{General procedure for the synthesis of $5 \mathbf{i}-\mathbf{m}$}

In the case of the synthesis of compounds $\mathbf{5 i}-\mathbf{m}, \mathrm{H}_{2} \mathrm{O}$ was used as the reaction medium and the round-bottom flask was fitted with a reflux condenser and heated with stirring in an oil-bath at reflux temperature for a period of time shown in Table 1.

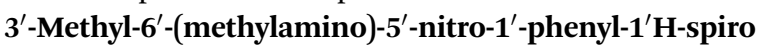
[indoline-3,4'-pyrano[2,3-c]pyrazol]-2-one (5a). White powder, mp: $268-272{ }^{\circ} \mathrm{C}$, yield $0.342 \mathrm{~g}(85 \%)$; IR $(\mathrm{KBr})\left(\nu_{\max } / \mathrm{cm}^{-1}\right): 3186$, $3060(\mathrm{NH}), 1686(\mathrm{C}=\mathrm{O}), 1532$ and $1386\left(\mathrm{NO}_{2}\right), 1223(\mathrm{C}-\mathrm{N}), 1015$ (C-O) 766 (Ar). ${ }^{1} \mathrm{H}$ NMR (300 MHz, DMSO): $\delta 1.58\left(3 \mathrm{H}, \mathrm{s}, \mathrm{CH}_{3}\right)$, $3.17\left(3 \mathrm{H}, \mathrm{d},{ }^{3} J_{\mathrm{HH}}=4.8 \mathrm{~Hz}, \mathrm{NCH}_{3}\right), 6.87-6.93(2 \mathrm{H}, \mathrm{m}, \mathrm{ArH}), 7.11$ $\left(1 \mathrm{H}, \mathrm{d},{ }^{3} J_{\mathrm{HH}}=7.2 \mathrm{~Hz}, \operatorname{ArH}\right), 7.18\left(1 \mathrm{H}, \mathrm{d},{ }^{3} J_{\mathrm{HH}}=7.5 \mathrm{~Hz}, \operatorname{ArH}\right)$, 7.34-7.39 (1H, m, ArH), 7.51-7.57 (2H, m, ArH), $7.73\left(2 \mathrm{H}, \mathrm{d},{ }^{3} J_{\mathrm{HH}}\right.$ $=8.1 \mathrm{~Hz}, \mathrm{ArH}), 10.71-10.73(1 \mathrm{H}, \mathrm{m}, \mathrm{NH}), 10.74(1 \mathrm{H}, \mathrm{s}, \mathrm{NH}) .{ }^{13} \mathrm{C}$ NMR (75.4 MHz, DMSO): $\delta 12.2\left(\mathrm{CH}_{3}\right), 29.4\left(\mathrm{CH}_{3} \mathrm{~N}\right), 50.0\left(\mathrm{C}_{\text {spiro }}\right)$, $98.3\left(\mathrm{C}-\mathrm{NO}_{2}\right), 107.5(\mathrm{C}=\mathrm{C}-\mathrm{N}), 109.7,121.1,121.2,122.4,122.6$, 123.6, 127.5, 128.9, 130.0, 130.1, 132.1, $137.3(\mathrm{Ar}), 142.9(\mathrm{C}=\mathrm{N})$, 144.7 (N-N-C-O), 159.4 (O-C-N), 176.6 (CO); MS (EI, $70 \mathrm{eV}): \mathrm{m} / \mathrm{z}$ $(\%)=403(9)[\mathrm{M}]^{+}, 387$ (32), 342 (78), 303 (67), 274 (67), 115 (35), 91 (55), 77 (100). Anal. calcd for $\mathrm{C}_{21} \mathrm{H}_{17} \mathrm{~N}_{5} \mathrm{O}_{4}$ (403.13): C, 62.53; H, 4.25; N, 17.36. Found C, 62.11; H, 3.90; N, 17.18.
5-Chloro-3' -methyl-6' -(methylamino)-5' -nitro-1' $^{\prime}$-phenyl-1' $\mathbf{H}^{-}$ spiro[indoline-3, $\mathbf{4}^{\prime}$-pyrano[2,3-c]pyrazol]-2-one $\quad(5 \mathrm{~b}) . \quad$ White powder, mp: $285-287{ }^{\circ} \mathrm{C}$, yield $0.349 \mathrm{~g}(80 \%)$; IR $(\mathrm{KBr})\left(\nu_{\max } /\right.$ $\left.\mathrm{cm}^{-1}\right)$ : 3372, $3025(\mathrm{NH}), 1686(\mathrm{C}=\mathrm{O}), 1498$ and $1363\left(\mathrm{NO}_{2}\right), 1213$ (C-N), 1102 (C-O) 776 (Ar), 585 (C-Cl). ${ }^{1} \mathrm{H}$ NMR $(300 \mathrm{MHz}$, DMSO): $\delta 1.65\left(3 \mathrm{H}, \mathrm{s}, \mathrm{CH}_{3}\right), 3.16\left(3 \mathrm{H}, \mathrm{d},{ }^{3} \mathrm{~J}_{\mathrm{HH}}=4.8 \mathrm{~Hz}, \mathrm{NCH}_{3}\right)$, $6.90\left(1 \mathrm{H}, \mathrm{d},{ }^{3} J_{\mathrm{HH}}=8.1 \mathrm{~Hz}, \operatorname{ArH}\right), 7.24\left(1 \mathrm{H}, \mathrm{d},{ }^{3} J_{\mathrm{HH}}=8.4 \mathrm{~Hz}, \mathrm{ArH}\right)$, 7.33-7.40 (1H, m, ArH), $7.34(1 \mathrm{H}, \mathrm{s}, \mathrm{ArH}), 7.52-7.57(2 \mathrm{H}, \mathrm{m}$, $\mathrm{ArH}), 7.72\left(2 \mathrm{H}, \mathrm{d},{ }^{3} \mathrm{~J}_{\mathrm{HH}}=8.1 \mathrm{~Hz}, \mathrm{ArH}\right), 10.74-10.76(1 \mathrm{H}, \mathrm{m}, \mathrm{NH})$, $10.90(1 \mathrm{H}, \mathrm{s}, \mathrm{NH}) .{ }^{13} \mathrm{C} \mathrm{NMR}(75.4 \mathrm{MHz}, \mathrm{DMSO}): \delta 12.1\left(\mathrm{CH}_{3}\right)$, $29.3\left(\mathrm{CH}_{3} \mathrm{~N}\right), 49.9\left(\mathrm{C}_{\text {spiro }}\right), 98.7\left(\mathrm{C}-\mathrm{NO}_{2}\right), 107.4(\mathrm{C}=\mathrm{C}-\mathrm{N}), 109.7$, 119.4 , 120.8, 122.4, 123.6, 127.2, 128.9, 131.8, 131.9, 134.3, 138.4, $142.9(\mathrm{Ar}), 143.2(\mathrm{C}=\mathrm{N}), 145.3(\mathrm{~N}-\mathrm{N}-\mathrm{C}-\mathrm{O}), 159.3(\mathrm{O}-\mathrm{C}-$ $\mathrm{N}), 176.4$ (CO). Anal. calcd for $\mathrm{C}_{21} \mathrm{H}_{16} \mathrm{ClN}_{5} \mathrm{O}_{4}$ (437.09): C, 57.61; H, 3.68; N, 16.00. Found C, 57.50; H, 3.92; N, 16.41.

5-Bromo-3' -methyl-6' -(methylamino)-5' -nitro-1' $^{\prime}$-phenyl-1' $\boldsymbol{H}^{-}$ spiro[indoline-3,4'-pyrano[2,3-c]pyrazol]-2-one $\quad(5 c)$. White powder, mp: $280-282{ }^{\circ} \mathrm{C}$, yield $0.375 \mathrm{~g}$ (78\%); IR (KBr) $\left(\nu_{\max } /\right.$ $\left.\mathrm{cm}^{-1}\right)$ : 3340, $3170(\mathrm{NH}), 1687(\mathrm{C}=\mathrm{O}), 1489$ and $1370\left(\mathrm{NO}_{2}\right), 1245$ (C-N), 1035 (C-O) 776 (Ar), 524 (C-Br). ${ }^{1} \mathrm{H}$ NMR (300 MHz, DMSO): $\delta 1.62\left(3 \mathrm{H}, \mathrm{s}, \mathrm{CH}_{3}\right), 3.16\left(3 \mathrm{H}, \mathrm{d},{ }^{3} \mathrm{~J}_{\mathrm{HH}}=5.1 \mathrm{~Hz}, \mathrm{NCH}_{3}\right)$, $6.85\left(1 \mathrm{H}, \mathrm{d},{ }^{3} J_{\mathrm{HH}}=8.1 \mathrm{~Hz}, \mathrm{ArH}\right), 7.32-7.42(3 \mathrm{H}, \mathrm{m}, \mathrm{ArH}), 7.46$ $(1 \mathrm{H}, \mathrm{s}, \mathrm{ArH}), 7.62\left(1 \mathrm{H}, \mathrm{d},{ }^{3} \mathrm{~J}_{\mathrm{HH}}=8.4 \mathrm{~Hz}, \mathrm{ArH}\right), 7.73\left(1 \mathrm{H}, \mathrm{d},{ }^{3} J_{\mathrm{HH}}=\right.$ 8.4 Hz, ArH), 10.74-10.76 (1H, m, NH), $10.90(1 \mathrm{H}, \mathrm{s}, \mathrm{NH}) .{ }^{13} \mathrm{C}$ NMR (75.4 MHz, DMSO): $\delta 12.1\left(\mathrm{CH}_{3}\right), 29.4\left(\mathrm{CH}_{3} \mathrm{~N}\right), 50.1\left(\mathrm{C}_{\text {spiro }}\right)$, $97.7\left(\mathrm{C}-\mathrm{NO}_{2}\right), 107.2(\mathrm{C}=\mathrm{C}-\mathrm{N}), 111.6,114.2,121.2,126.7,127.6$, 129.4, 130.1, 131.6, 131.8, 134.6, 137.2, 142.2 (Ar), $143.1(\mathrm{C}=\mathrm{N})$, $144.5(\mathrm{~N}-\mathrm{N}-\mathrm{C}-\mathrm{O}), 159.4(\mathrm{O}-\mathrm{C}-\mathrm{N}), 176.2$ (CO).

$\mathbf{1}^{\prime}$-(3-Chlorophenyl)-3' -methyl-6' -(methylamino)-5' -nitro-1' $\mathrm{H}^{\prime}$ spiro[indoline-3, $\mathbf{4}^{\prime}$-pyrano[2,3-c]pyrazol]-2-one (5d). White powder, mp: $272-275{ }^{\circ} \mathrm{C}$, yield $0.375 \mathrm{~g}$ (86\%); IR (KBr) $\left(\nu_{\max } /\right.$ $\left.\mathrm{cm}^{-1}\right)$ : 3130, $3052(\mathrm{OH}), 1701(\mathrm{C}=\mathrm{O}), 1584$ and $1397\left(\mathrm{NO}_{2}\right), 1217$ (C-N), 1026 (C-O) 772 (Ar), 542 (C-Cl). ${ }^{1} \mathrm{H}$ NMR $(300 \mathrm{MHz}$, DMSO): $\delta 1.60\left(3 \mathrm{H}, \mathrm{s}, \mathrm{CH}_{3}\right), 3.17\left(3 \mathrm{H}, \mathrm{d},{ }^{3} \mathrm{~J}_{\mathrm{HH}}=6.3 \mathrm{~Hz}, \mathrm{NCH}_{3}\right)$, 6.87-6.93 (2H, m, ArH), $7.10\left(1 \mathrm{H}, \mathrm{d},{ }^{3} J_{\mathrm{HH}}=7.2 \mathrm{~Hz}, \mathrm{ArH}\right), 7.17-$ $7.22(1 \mathrm{H}, \mathrm{m}, \mathrm{ArH}), 7.43\left(1 \mathrm{H}, \mathrm{d},{ }^{3} J_{\mathrm{HH}}=8.1 \mathrm{~Hz}, \operatorname{ArH}\right), 7.57(1 \mathrm{H}, \mathrm{d}$, $\left.{ }^{3} J_{\mathrm{HH}}=8.1 \mathrm{~Hz}, \mathrm{ArH}\right), 7.78\left(1 \mathrm{H}, \mathrm{d},{ }^{3} J_{\mathrm{HH}}=7.2 \mathrm{~Hz}, \operatorname{ArH}\right), 7.84(1 \mathrm{H}, \mathrm{s}$, ArH), 10.70-10.72 (1H, m, NH), 10.75 (1H, s, NH). ${ }^{13} \mathrm{C}$ NMR (75.4 MHz, DMSO): $\delta 12.2\left(\mathrm{CH}_{3}\right), 29.4\left(\mathrm{CH}_{3} \mathrm{~N}\right), 50.2\left(\mathrm{C}_{\text {spiro }}\right), 97.7(\mathrm{C}-$ $\left.\mathrm{NO}_{2}\right), 107.2(\mathrm{C}=\mathrm{C}-\mathrm{N}), 111.1,121.1,121.2,124.0,126.5,127.6$, 128.7, 130.0, 130.1, 134.2, $141.8(\mathrm{Ar}), 143.1(\mathrm{C}=\mathrm{N}), 144.5(\mathrm{~N}-\mathrm{N}-$ C-O), 159.4 (O-C-N), 176.4 (CO); MS (EI, $70 \mathrm{eV}): \mathrm{m} / z(\%)=438$ (1) $[\mathrm{M}+1]^{+}, 437(3)[\mathrm{M}]^{+}, 391$ (25), 376 (100), 363 (23), 209 (24), $111(32)$.

5-Bromo-1' -(3-chlorophenyl)-3' -methyl-6' -(methylamino)-5' nitro-1' ${ }^{\prime}$-spiro[indoline-3,4'-pyrano[2,3-c]pyrazol]-2-one (5e). White powder, mp: $268-271{ }^{\circ} \mathrm{C}$, yield $0.386 \mathrm{~g}(75 \%) .{ }^{1} \mathrm{H}$ NMR (300 MHz, DMSO): $\delta 1.62\left(3 \mathrm{H}, \mathrm{s}, \mathrm{CH}_{3}\right), 3.26\left(3 \mathrm{H}, \mathrm{d},{ }^{3} J_{\mathrm{HH}}=\right.$ $\left.4.5 \mathrm{~Hz}, \mathrm{NCH}_{3}\right), 6.85\left(1 \mathrm{H}, \mathrm{d},{ }^{3} J_{\mathrm{HH}}=7.8 \mathrm{~Hz}, \mathrm{ArH}\right), 7.38\left(1 \mathrm{H}, \mathrm{d},{ }^{3} J_{\mathrm{HH}}\right.$ $=8.1 \mathrm{~Hz}, \mathrm{ArH}), 7.45\left(1 \mathrm{H}, \mathrm{d},{ }^{3} J_{\mathrm{HH}}=8.1 \mathrm{~Hz}, \operatorname{ArH}\right), 7.50(1 \mathrm{H}, \mathrm{s}$, ArH), 7.56-7.61 (1H, m, ArH), $7.72\left(1 \mathrm{H}, \mathrm{d},{ }^{3} \mathrm{~J}_{\mathrm{HH}}=8.1 \mathrm{~Hz}, \operatorname{ArH}\right)$, 7.83 (1H, s, ArH), 10.74-10.83 (1H, m, NH), 10.90 (1H, s, NH). ${ }^{13} \mathrm{C}$ NMR (75.4 MHz, DMSO): $\delta 12.2\left(\mathrm{CH}_{3}\right), 29.4\left(\mathrm{CH}_{3} \mathrm{~N}\right), 50.0$ $\left(\mathrm{C}_{\text {spiro }}\right), 98.2\left(\mathrm{C}-\mathrm{NO}_{2}\right), 107.2(\mathrm{C}=\mathrm{C}-\mathrm{N}), 111.7,114.2,119.4,120.8$, 126.7, 127.2, 131.7, 131.8, 134.3, 134.4, 138.3, 142.2 (Ar), 143.4 $(\mathrm{C}=\mathrm{N}), 145.2(\mathrm{~N}-\mathrm{N}-\mathrm{C}-\mathrm{O}), 159.3(\mathrm{O}-\mathrm{C}-\mathrm{N}), 176.1(\mathrm{CO}) ; \mathrm{MS}(\mathrm{EI}, 70$ 
$\mathrm{eV}): m / z(\%)=517(6)[\mathrm{M}+2]^{+}, 515(5)[\mathrm{M}]^{+}, 501(24), 456(100)$, 342 (38), 125 (46), 111 (84).

5-Chloro-1' -(3-chlorophenyl)-3' -methyl-6' -(methylamino)-5' nitro-1' $\boldsymbol{H}$-spiro[indoline-3,4'-pyrano[2,3-c]pyrazol]-2-one (5f). White powder, mp: $254-258{ }^{\circ} \mathrm{C}$, yield $0.376 \mathrm{~g}(80 \%) .{ }^{1} \mathrm{H}$ NMR (300 MHz, DMSO): $\delta 1.65\left(3 \mathrm{H}, \mathrm{s}, \mathrm{CH}_{3}\right), 3.18\left(3 \mathrm{H}, \mathrm{d},{ }^{3} \mathrm{~J}_{\mathrm{HH}}=\right.$ $\left.3.6 \mathrm{~Hz}, \mathrm{NCH}_{3}\right), 6.90\left(1 \mathrm{H}, \mathrm{d},{ }^{3} J_{\mathrm{HH}}=8.4 \mathrm{~Hz}, \mathrm{ArH}\right), 7.25\left(1 \mathrm{H}, \mathrm{d},{ }^{3} J_{\mathrm{HH}}\right.$ $=8.1 \mathrm{~Hz}, \operatorname{ArH}), 7.32(1 \mathrm{H}, \mathrm{s}, \operatorname{ArH}), 7.45\left(1 \mathrm{H}, \mathrm{d},{ }^{3} J_{\mathrm{HH}}=7.5 \mathrm{~Hz}\right.$, ArH), 7.55-7.61 (1H, m, ArH), $7.71\left(1 \mathrm{H}, \mathrm{d},{ }^{3} J_{\mathrm{HH}}=8.4 \mathrm{~Hz}, \mathrm{ArH}\right)$, 7.83 (1H, s, ArH), 10.68-10.79 (1H, m, NH), 10.89 (1H, s, NH). ${ }^{13} \mathrm{C}$ NMR (75.4 MHz, DMSO): $\delta 12.2\left(\mathrm{CH}_{3}\right), 29.4\left(\mathrm{CH}_{3} \mathrm{~N}\right), 50.0$ $\left(\mathrm{C}_{\text {spiro }}\right), 98.1\left(\mathrm{C}-\mathrm{NO}_{2}\right), 107.2(\mathrm{C}=\mathrm{C}-\mathrm{N}), 111.1,119.5,120.9,124.0$, 126.5, 127.3, 128.8, 131.8, 134.1, 134.3, 138.3, 141.8 (Ar), 143.4 $(\mathrm{C}=\mathrm{N}), 145.2(\mathrm{~N}-\mathrm{N}-\mathrm{C}-\mathrm{O}), 159.3(\mathrm{O}-\mathrm{C}-\mathrm{N}), 176.2$ (CO); MS (EI, 70 $\mathrm{eV}): m / z(\%)=472(5)[\mathrm{M}+1]^{+}, 471(7)[\mathrm{M}]^{+}, 425(20), 410(100)$, 336 (17), 192 (22), 166 (24), 125 (33), 111 (66).

5-Bromo-1' -methyl-6' $^{\prime}$-(methylamino)-5' -nitro-3' -(trifluoromethyl)-1' $H$-spiro[indoline-3, $4^{\prime}$-pyrano[2,3-c]pyrazol]-2one (5g). White powder, mp: $298-301{ }^{\circ} \mathrm{C}$, yield $0.292 \mathrm{~g}(62 \%) .{ }^{1} \mathrm{H}$ NMR (300 MHz, DMSO): $\delta 3.20\left(3 \mathrm{H}, \mathrm{d},{ }^{3} J_{\mathrm{HH}}=3.6 \mathrm{~Hz}, \mathrm{NHCH}_{3}\right)$, $3.86\left(3 \mathrm{H}, \mathrm{s}, \mathrm{NCH}_{3}\right), 7.04\left(1 \mathrm{H}, \mathrm{d},{ }^{3} J_{\mathrm{HH}}=7.2 \mathrm{~Hz}, \mathrm{ArH}\right), 7.34(1 \mathrm{H}, \mathrm{d}$, $\left.{ }^{3} J_{\mathrm{HH}}=7.5 \mathrm{~Hz}, \mathrm{ArH}\right), 7.39(1 \mathrm{H}, \mathrm{s}, \mathrm{ArH}), 10.71-10.76(1 \mathrm{H}, \mathrm{m}, \mathrm{NH})$, $10.80(1 \mathrm{H}, \mathrm{s}, \mathrm{NH}) .{ }^{13} \mathrm{C} \mathrm{NMR}(75.4 \mathrm{MHz}, \mathrm{DMSO}): \delta 29.2\left(\mathrm{CH}_{3}\right)$, $35.6\left(\mathrm{CH}_{3} \mathrm{~N}\right), 49.9\left(\mathrm{C}_{\text {spiro }}\right), 96.1\left(\mathrm{C}-\mathrm{NO}_{2}\right), 107.5(\mathrm{C}=\mathrm{C}-\mathrm{N}), 125.0$, 111.5, 113.6, 126.6, 131.7, 134.5, $142.7(\mathrm{Ar}), 134.0(\mathrm{C}=\mathrm{N}), 145.2$ (N-N-C-O), 158.5 (O-C-N), 176.1 (CO).

6'-(Benzylamino)-5-chloro-3' ${ }^{\prime}$-methyl-5' ${ }^{\prime}$ nitro-1' -phenyl-1' $^{\prime} \boldsymbol{H}$ spiro[indoline-3,4'-pyrano[2,3-c]pyrazol]-2-one (5h). Red powder, mp: $271-274{ }^{\circ} \mathrm{C}$, yield $0.328 \mathrm{~g}$ (58\%). ${ }^{1} \mathrm{H}$ NMR (300 MHz, DMSO): $\delta 3.10\left(3 \mathrm{H}, \mathrm{d},{ }^{3} J_{\mathrm{HH}}=4.2 \mathrm{~Hz}, \mathrm{NHCH}_{3}\right), 3.33-3.45$ $\left(2 \mathrm{H}, \mathrm{m}, \mathrm{CH}_{2}\right), 7.14-7.64$ (8H, m, ArH), 11.20-11.31 (1H, m, NH), $12.53(1 \mathrm{H}, \mathrm{s}, \mathrm{NH}) .{ }^{13} \mathrm{C} \mathrm{NMR}(75.4 \mathrm{MHz}, \mathrm{DMSO}): \delta 25.6\left(\mathrm{CH}_{2}\right)$, $47.9\left(\mathrm{C}_{\text {spiro }}\right), 99.2\left(\mathrm{C}-\mathrm{NO}_{2}\right), 117.0(\mathrm{C}=\mathrm{C}-\mathrm{N}), 119.8,120.2,121.0$, 123.5, 130.7, 131.5, 132.2, 132.6, 134.2, 135.7, 136.7 (Ar), 149.9 $(\mathrm{C}=\mathrm{N}), 156.9(\mathrm{~N}-\mathrm{N}-\mathrm{C}-\mathrm{O}), 164.7(\mathrm{O}-\mathrm{C}-\mathrm{N}), 176.0(\mathrm{CO})$.

5-Chloro-7' -(methylamino)-6' -nitrospiro[indoline-3, $5^{\prime}$-pyrano[2,3-d] pyrimidine $]-2,2^{\prime}, \mathbf{4}^{\prime}\left(\mathbf{1}^{\prime} \boldsymbol{H}, 3^{\prime} \boldsymbol{H}\right)$-trione (5i). White powder, mp: $287-293{ }^{\circ} \mathrm{C}$, yield $0.254 \mathrm{~g}(65 \%)$; IR (KBr) $\left(\nu_{\max } /\right.$ $\left.\mathrm{cm}^{-1}\right): 3410,3196(\mathrm{NH}), 1730,1653(\mathrm{C}=\mathrm{O}), 1472$ and 1371 $\left(\mathrm{NO}_{2}\right), 1241(\mathrm{C}-\mathrm{N}), 1126(\mathrm{C}-\mathrm{O}) 768(\mathrm{Ar}), 560(\mathrm{C}-\mathrm{Cl}) .{ }^{1} \mathrm{H}$ NMR $(300$ MHz, DMSO): $\delta 3.08\left(3 \mathrm{H}, \mathrm{d},{ }^{3} J_{\mathrm{HH}}=4.8 \mathrm{~Hz}, \mathrm{NCH}_{3}\right), 6.96(1 \mathrm{H}, \mathrm{d}$, $\left.{ }^{3} J_{\mathrm{HH}}=8.1 \mathrm{~Hz}, \mathrm{ArH}\right), 7.11\left(1 \mathrm{H}, \mathrm{d},{ }^{3} J_{\mathrm{HH}}=8.1 \mathrm{~Hz}, \operatorname{ArH}\right), 7.34(1 \mathrm{H}, \mathrm{s}$, ArH), 10.51-10.53 (1H, m, NH), 10.66 (1H, s, NH), 11.23 (1H, s, $\mathrm{NH}), 12.52$ (1H, s, NH). ${ }^{13} \mathrm{C}$ NMR (75.4 MHz, DMSO): $\delta 29.3$ $\left(\mathrm{CH}_{3} \mathrm{~N}\right), 48.7\left(\mathrm{C}_{\text {spiro }}\right), 88.9\left(\mathrm{C}-\mathrm{NO}_{2}\right), 107.5(\mathrm{C}=\mathrm{C}-\mathrm{O}), 110.1,123.8$, 125.4, 128.3, 133.2, 143.9 (Ar), 149.4 (NCON), $152.0(\mathrm{~N}-\mathrm{C}-\mathrm{O})$, $157.0\left(\mathrm{O}-\mathrm{C}-\mathrm{NCH}_{3}\right), 161.3(\mathrm{CO}), 176.6(\mathrm{CO})$. Anal. calcd for $\mathrm{C}_{15} \mathrm{H}_{10} \mathrm{ClN}_{5} \mathrm{O}_{6}$ (391.03): C, 45.99; H, 2.57; N, 17.88. Found C, 46.11; H, 2.38; N, 18.01 .

5-Chloro-6' -nitro-7' $^{\prime}$-(propylamino)spiro[indoline-3,5' -pyrano [2,3-d]pyrimidine]-2,2', $\mathbf{4}^{\prime}\left(\mathbf{1}^{\prime} \boldsymbol{H}, \mathbf{3}^{\prime} \boldsymbol{H}\right)$-trione (5j). White powder, mp: $240-242{ }^{\circ} \mathrm{C}$, yield $0.314 \mathrm{~g}(75 \%)$; IR (KBr) $\left(\nu_{\max } / \mathrm{cm}^{-1}\right): 3393$, $3140(\mathrm{NH}), 1702(\mathrm{C}=\mathrm{O}), 1607(\mathrm{C}=\mathrm{O}), 1472$ and $1372\left(\mathrm{NO}_{2}\right)$, $1179(\mathrm{C}-\mathrm{N}), 1040$ (C-O), 599 (C-Cl). ${ }^{1} \mathrm{H}$ NMR (300 MHz, DMSO): $\delta 0.87\left(3 \mathrm{H}, \mathrm{t},{ }^{3} J_{\mathrm{HH}}=7.8 \mathrm{~Hz}, \mathrm{CH}_{3}\right), 1.44-1.57\left(2 \mathrm{H}, \mathrm{m}, \mathrm{CH}_{2}\right), 2.68-$ $2.74\left(2 \mathrm{H}, \mathrm{m}, \mathrm{NCH}_{2}\right), 6.60\left(1 \mathrm{H}, \mathrm{d},{ }^{3} J_{\mathrm{HH}}=8.1 \mathrm{~Hz}, \mathrm{ArH}\right), 6.92(1 \mathrm{H}, \mathrm{s}$, $\mathrm{ArH}), 7.03\left(1 \mathrm{H}, \mathrm{d},{ }^{3} \mathrm{~J}_{\mathrm{HH}}=8.1 \mathrm{~Hz}, \operatorname{ArH}\right), 7.41-7.69(1 \mathrm{H}, \mathrm{m}, \mathrm{NH})$,
$9.31(1 \mathrm{H}, \mathrm{s}, \mathrm{NH}), 9.80(1 \mathrm{H}, \mathrm{s}, \mathrm{NH}), 9.90(1 \mathrm{H}, \mathrm{s}, \mathrm{NH}) .{ }^{13} \mathrm{C} \mathrm{NMR}$ (75.4 MHz, DMSO): $\delta 11.3\left(\mathrm{CH}_{3}\right), 20.8\left(\mathrm{CH}_{2}\right), 41.0\left(\mathrm{NCH}_{2}\right), 56.5$ $\left(\mathrm{C}_{\text {spiro }}\right), 77.2\left(\mathrm{C}-\mathrm{NO}_{2}\right), 84.0(\mathrm{C}=\mathrm{C}-\mathrm{O}), 110.5,123.3,125.2,126.1$, 127.6, 130.1 (Ar), 139.3 (NCON), 141.6 (N-C-O), 151.9 (O-C$\mathrm{NCH}_{3}$ ), 152.2 (CO), 179.5 (CO); MS (EI, $\left.70 \mathrm{eV}\right): m / z(\%)=419$ (0.01) [M] $]^{+}, 181$ (47), 153 (100), 128 (53), 90 (19), 63 (40).

5-Chloro- $7^{\prime}$-(isopropylamino)- $6^{\prime}$-nitrospiro[indoline-3, $\mathbf{5}^{\prime}$-pyrano[2,3-d]pyrimidine $]-2,2^{\prime}, \mathbf{4}^{\prime}\left(\mathbf{1}^{\prime} \boldsymbol{H}, \mathbf{3}^{\prime} \boldsymbol{H}\right)$-trione (5k). White powder, mp: $270-272{ }^{\circ} \mathrm{C}$, yield $0.318 \mathrm{~g}$ (76\%); IR (KBr) $\left(\nu_{\max } /\right.$ $\left.\mathrm{cm}^{-1}\right)$ : 3161, $3046(\mathrm{NH}), 1729,1650,1601(\mathrm{C}=\mathrm{O}), 1466$ and 1373 $\left(\mathrm{NO}_{2}\right), 1239(\mathrm{C}-\mathrm{N}), 1021(\mathrm{C}-\mathrm{O}) 758(\mathrm{Ar}), 550(\mathrm{C}-\mathrm{Cl}) .{ }^{1} \mathrm{H}$ NMR $(300$ MHz, DMSO): $\delta 1.12\left(6 \mathrm{H}, \mathrm{d},{ }^{3} J_{\mathrm{HH}}=6.6 \mathrm{~Hz}, \mathrm{CH}_{3}\right), 3.19-3.28(1 \mathrm{H}$, $\mathrm{m}, \mathrm{CH}), 6.61\left(1 \mathrm{H}, \mathrm{d},{ }^{3} \mathrm{~J}_{\mathrm{HH}}=8.1 \mathrm{~Hz}, \mathrm{ArH}\right), 6.92(1 \mathrm{H}, \mathrm{s}, \mathrm{ArH}), 7.03$ $\left(1 \mathrm{H}, \mathrm{d},{ }^{3} \mathrm{~J}_{\mathrm{HH}}=8.1 \mathrm{~Hz}, \mathrm{ArH}\right), 7.42-7.68(1 \mathrm{H}, \mathrm{m}, \mathrm{NH}), 9.36(1 \mathrm{H}, \mathrm{s}$, $\mathrm{NH}), 9.82(1 \mathrm{H}, \mathrm{s}, \mathrm{NH}), 9.88(1 \mathrm{H}, \mathrm{s}, \mathrm{NH}) .{ }^{13} \mathrm{C} \mathrm{NMR}(75.4 \mathrm{MHz}$, DMSO): $\delta 20.8\left(\mathrm{CH}_{3}\right), 43.5(\mathrm{CH}), 77.2\left(\mathrm{C}-\mathrm{NO}_{2}\right), 83.9(\mathrm{C}=\mathrm{C}-\mathrm{O})$, 110.5, 123.3, 125.2, $127.6(\mathrm{Ar}), 139.3(\mathrm{NCON}), 141.6(\mathrm{~N}-\mathrm{C}-\mathrm{O})$, $152.2\left(\mathrm{O}-\mathrm{C}-\mathrm{NCH}_{3}\right), 152.2$ (CO), 179.5 (CO); MS (EI, $\left.70 \mathrm{eV}\right): \mathrm{m} / \mathrm{z}$ $(\%)=419$ (0.01) $[\mathrm{M}]^{+}, 181$ (48), 153 (100), 128 (53), 110 (4), 90 (18), 63 (36).

5-Bromo-6'-nitro-7' -(propylamino)spiro[indoline-3,5' -pyrano [2,3-d]pyrimidine $]-2, \mathbf{2}^{\prime}, \mathbf{4}^{\prime}\left(\mathbf{1}^{\prime} \boldsymbol{H}, \mathbf{3}^{\prime} \boldsymbol{H}\right)$-trione (5l). White powder, mp: $235-239{ }^{\circ} \mathrm{C}$, yield $0.328 \mathrm{~g}(71 \%) .{ }^{1} \mathrm{H}$ NMR (300 MHz, DMSO): $\delta 0.90\left(3 \mathrm{H}, \mathrm{t},{ }^{3} J_{\mathrm{HH}}=8.1 \mathrm{~Hz}, \mathrm{CH}_{3}\right), 1.47-1.54\left(2 \mathrm{H}, \mathrm{m}, \mathrm{CH}_{2}\right), 2.65-$ $2.73\left(2 \mathrm{H}, \mathrm{m}, \mathrm{NCH}_{2}\right), 6.57\left(1 \mathrm{H}, \mathrm{d},{ }^{3} J_{\mathrm{HH}}=8.1 \mathrm{~Hz}, \mathrm{ArH}\right), 7.03(1 \mathrm{H}, \mathrm{s}$, ArH), $7.16\left(1 \mathrm{H}, \mathrm{d},{ }^{3} \mathrm{~J}_{\mathrm{HH}}=8.1 \mathrm{~Hz}, \mathrm{ArH}\right), 7.38-7.78(1 \mathrm{H}, \mathrm{m}, \mathrm{NH})$, $9.30(1 \mathrm{H}, \mathrm{s}, \mathrm{NH}), 9.81(1 \mathrm{H}, \mathrm{s}, \mathrm{NH}), 9.90(1 \mathrm{H}, \mathrm{s}, \mathrm{NH}) .{ }^{13} \mathrm{C} \mathrm{NMR}$ (75.4 MHz, DMSO): $\delta 11.3\left(\mathrm{CH}_{3}\right), 20.9\left(\mathrm{CH}_{2}\right), 41.0\left(\mathrm{NCH}_{2}\right), 56.7$ $\left(\mathrm{C}_{\text {spiro }}\right), 77.2\left(\mathrm{C}-\mathrm{NO}_{2}\right), 83.8(\mathrm{C}=\mathrm{C}-\mathrm{O}), 111.0,112.8,125.5,126.0$, 130.4 (Ar), 139.9 (NCON), $142.0(\mathrm{~N}-\mathrm{C}-\mathrm{O}), 143.8\left(\mathrm{O}-\mathrm{C}-\mathrm{NCH}_{3}\right)$, 152.1 (CO), 179.3 (CO).

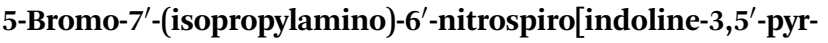
ano[2,3- $\boldsymbol{d}]$ pyrimidine $]-2,2^{\prime}, \mathbf{4}^{\prime}\left(\mathbf{1}^{\prime} \boldsymbol{H}, \mathbf{3}^{\prime} \boldsymbol{H}\right)$-trione $\quad(5 \mathrm{~m})$. White powder, mp: 260-262 ${ }^{\circ} \mathrm{C}$, yield $0.333 \mathrm{~g}$ (72\%). ${ }^{1} \mathrm{H}$ NMR (300 MHz, DMSO): $\delta 1.12\left(6 \mathrm{H}, \mathrm{d},{ }^{3} J_{\mathrm{HH}}=6.3 \mathrm{~Hz}, \mathrm{CH}_{3}\right), 3.21-3.33(1 \mathrm{H}$, $\mathrm{m}, \mathrm{CH}), 6.67\left(1 \mathrm{H}, \mathrm{d},{ }^{3} J_{\mathrm{HH}}=8.1 \mathrm{~Hz}, \mathrm{ArH}\right), 7.03(1 \mathrm{H}, \mathrm{s}, \mathrm{ArH}), 7.16$ $\left(1 \mathrm{H}, \mathrm{d},{ }^{3} J_{\mathrm{HH}}=8.1 \mathrm{~Hz}, \mathrm{ArH}\right), 7.55-7.81(1 \mathrm{H}, \mathrm{m}, \mathrm{NH}), 9.32(1 \mathrm{H}, \mathrm{s}$, $\mathrm{NH}), 9.82(1 \mathrm{H}, \mathrm{s}, \mathrm{NH}), 9.89(1 \mathrm{H}, \mathrm{s}, \mathrm{NH}) .{ }^{13} \mathrm{C} \mathrm{NMR}(75.4 \mathrm{MHz}$, DMSO): $\delta 20.8\left(\mathrm{CH}_{3}\right), 43.5(\mathrm{CH}), 44.3\left(\mathrm{C}_{\text {spiro }}\right), 77.2\left(\mathrm{C}-\mathrm{NO}_{2}\right), 83.9$ $(\mathbf{C}=\mathrm{C}-\mathrm{O}), 111.1,112.9,117.2,124.0,126.1,130.4(\mathrm{Ar}), 139.7$ (NCON), 142.0 (N-C-O), $152.1\left(\mathrm{O}-\mathrm{C}-\mathrm{NCH}_{3}\right), 162.0$ (CO), 179.3 (CO).

25'-Chloro-2-(methylamino)-3-nitro-5 $\mathrm{H}$-spiro[indeno[1,2-b] pyran-4,3'-indoline]-2',5-dione (5n). Red powder, mp: 238$240{ }^{\circ} \mathrm{C}$, yield $0.265 \mathrm{~g}(65 \%) .{ }^{1} \mathrm{H}$ NMR (300 MHz, DMSO): $\delta 3.39$ $\left(3 \mathrm{H}, \mathrm{d},{ }^{3} \mathrm{~J}_{\mathrm{HH}}=7.2 \mathrm{~Hz}, \mathrm{CH}_{3}\right), 7.11-7.63(7 \mathrm{H}, \mathrm{m}, \mathrm{ArH}), 11.21-11.32$ (1H, m, NH), 12.53 (1H, s, NH). ${ }^{13} \mathrm{C}$ NMR (75.4 MHz, DMSO): $\delta 25.6\left(\mathrm{CH}_{3}\right), 48.0\left(\mathrm{C}_{\text {spiro }}\right), 89.8\left(\mathrm{C}-\mathrm{NO}_{2}\right), 99.1(\mathrm{C}=\mathrm{C}-\mathrm{O}), 119.7$, 120.2, 120.9, 127.9, 129.0, 131.5, 132.2, 134.5, 135.8, 136.3, 149.8 (Ar), $156.9(\mathrm{O}-\mathrm{C}-\mathrm{N}), 164.7$ (C=C-O), 174.9 (NCO), 188.6 (CO).

2-(Benzylamino)-3-nitrospiro[benzo $[\mathrm{g}]$ chromene-4, $\mathbf{3}^{\prime}$-indoline]-2 ${ }^{\prime}, \mathbf{5 , 1 0}$-trione (5o). Red powder, mp: $>300{ }^{\circ} \mathrm{C}$, yield $0.311 \mathrm{~g}$ (65\%). ${ }^{1} \mathrm{H}$ NMR (300 MHz, DMSO): $\delta$ 4.79-4.89 $\left(2 \mathrm{H}, \mathrm{m}, \mathrm{CH}_{2}\right)$, 6.76-6.81 (1H, m, ArH), $7.13\left(1 \mathrm{H}, \mathrm{d},{ }^{3} J_{\mathrm{HH}}=6.6 \mathrm{~Hz}, \mathrm{ArH}\right), 7.32$ $\left(1 \mathrm{H}, \mathrm{d},{ }^{3} J_{\mathrm{HH}}=6.3 \mathrm{~Hz}, \mathrm{ArH}\right), 7.37-7.41(1 \mathrm{H}, \mathrm{m}, \mathrm{ArH}), 7.58(1 \mathrm{H}, \mathrm{d}$, $\left.{ }^{3} J_{\mathrm{HH}}=7.5 \mathrm{~Hz}, \mathrm{ArH}\right), 7.71-7.84(2 \mathrm{H}, \mathrm{m}, \mathrm{ArH}), 8.05\left(1 \mathrm{H}, \mathrm{d},{ }^{3} J_{\mathrm{HH}}=\right.$ $6.9 \mathrm{~Hz}, \mathrm{ArH}), 10.74$ (1H, s, NH), 10.92-11.03 (1H, m, NH). ${ }^{13} \mathrm{C}$ 
NMR (75.4 MHz, DMSO): $\delta 45.6\left(\mathrm{CH}_{3}\right), 50.2\left(\mathrm{C}_{\text {spiro }}\right), 107.5(\mathrm{C}-$ $\left.\mathrm{NO}_{2}\right), 109.4(\mathrm{C}=\mathrm{C}-\mathrm{O}), 121.7,124.1,126.5,126.8,128.2$, 129.1, 129.3, 131.2 , 131.3, 135.4, 137.9, 144.7 (Ar), $148.6(\mathrm{O}-\mathrm{C}-\mathrm{N}), 156.3$ $(\mathrm{C}=\mathrm{C}-\mathrm{O}), 176.2$ (NCO), 176.5 (CO), 181.4 (CO).

5 '-Chloro-3-nitro-2-(propylamino)spiro[benzo $[g]$ chromene4,3'-indoline]-2',5,10-trione (5p). Red powder, $\mathrm{mp}:>300{ }^{\circ} \mathrm{C}$, yield $0.288 \mathrm{~g}(62 \%)$; IR (KBr) $\left(\nu_{\max } / \mathrm{cm}^{-1}\right): 3214,3061(\mathrm{NH}), 1731$, 1651, $1605(\mathrm{C}=\mathrm{O}), 1524$ and $1352\left(\mathrm{NO}_{2}\right), 1214(\mathrm{C}-\mathrm{N}), 1067(\mathrm{C}-\mathrm{O})$ $768(\mathrm{Ar}), 542(\mathrm{C}-\mathrm{Cl}) .{ }^{1} \mathrm{H}$ NMR (300 MHz, DMSO): $\delta 0.85(3 \mathrm{H}, \mathrm{t}$, $\left.{ }^{3} J_{\mathrm{HH}}=8.1 \mathrm{~Hz}, \mathrm{CH}_{3}\right), 1.50-1.60\left(2 \mathrm{H}, \mathrm{m}, \mathrm{CH}_{2}\right), 2.62-2.77(2 \mathrm{H}, \mathrm{m}$, $\left.\mathrm{NCH}_{2}\right), 6.88\left(1 \mathrm{H}, \mathrm{d},{ }^{3} J_{\mathrm{HH}}=8.4 \mathrm{~Hz}, \operatorname{ArH}\right), 7.30\left(1 \mathrm{H}, \mathrm{d},{ }^{3} J_{\mathrm{HH}}=\right.$ $8.4 \mathrm{~Hz}, \mathrm{ArH}), 7.41(1 \mathrm{H}, \mathrm{s}, \mathrm{ArH}), 7.60\left(1 \mathrm{H}, \mathrm{d},{ }^{3} J_{\mathrm{HH}}=7.8 \mathrm{~Hz}, \operatorname{ArH}\right)$, 7.67-7.72 (2H, m, ArH), $7.82\left(1 \mathrm{H}, \mathrm{d},{ }^{3} \mathrm{~J}_{\mathrm{HH}}=7.8 \mathrm{~Hz}, \mathrm{ArH}\right), 10.28-$ $10.67(1 \mathrm{H}, \mathrm{m}, \mathrm{NH}), 10.89(1 \mathrm{H}, \mathrm{s}, \mathrm{NH}) .{ }^{13} \mathrm{C} \mathrm{NMR}(75.4 \mathrm{MHz}$, DMSO): $\delta 11.5\left(\mathrm{CH}_{3}\right), 21.1\left(\mathrm{CH}_{2}\right), 41.2\left(\mathrm{NCH}_{2}\right), 55.0\left(\mathrm{C}_{\text {spiro }}\right), 104.2$ $\left(\mathrm{C}-\mathrm{NO}_{2}\right), 112.7(\mathrm{C}=\mathrm{C}-\mathrm{O}), 125.7,126.1,128.1,131.4,131.8$, 133.4, 134.5 (Ar), $147.6(\mathrm{O}-\mathrm{C}-\mathrm{N}), 155.5(\mathrm{C}=\mathrm{C}-\mathrm{O}), 176.5$ (NCO), 177.0 (CO), 182.0 (CO).

$5^{\prime}$-Bromo-3-nitro-2-(propylamino)spiro[benzo $[g]$ chromene-4, $3^{\prime}$ indoline]-2',5,10-trione (5q). Red powder, mp: $>300{ }^{\circ} \mathrm{C}$, yield $0.305 \mathrm{~g}(60 \%) .{ }^{1} \mathrm{H}$ NMR (300 MHz, DMSO): $\delta 0.82\left(3 \mathrm{H}, \mathrm{t},{ }^{3} J_{\mathrm{HH}}=\right.$ $\left.8.4 \mathrm{~Hz}, \mathrm{CH}_{3}\right), 1.50-1.60\left(2 \mathrm{H}, \mathrm{m}, \mathrm{CH}_{2}\right), 2.62-2.80\left(2 \mathrm{H}, \mathrm{m}, \mathrm{NCH}_{2}\right)$, $6.83\left(1 \mathrm{H}, \mathrm{d},{ }^{3} J_{\mathrm{HH}}=8.4 \mathrm{~Hz}, \mathrm{ArH}\right), 7.43\left(1 \mathrm{H}, \mathrm{d},{ }^{3} J_{\mathrm{HH}}=8.4 \mathrm{~Hz}, \operatorname{ArH}\right)$, $7.53(1 \mathrm{H}, \mathrm{s}, \mathrm{ArH}), 7.55\left(1 \mathrm{H}, \mathrm{d},{ }^{3} J_{\mathrm{HH}}=7.5 \mathrm{~Hz}, \mathrm{ArH}\right), 7.62-7.72(2 \mathrm{H}$, $\mathrm{m}, \mathrm{ArH}), 7.82\left(1 \mathrm{H}, \mathrm{d},{ }^{3} J_{\mathrm{HH}}=7.2 \mathrm{~Hz}, \mathrm{ArH}\right), 10.28-10.65(1 \mathrm{H}, \mathrm{m}, \mathrm{NH})$, 10.90 (1H, s, NH). ${ }^{13} \mathrm{C}$ NMR (75.4 MHz, DMSO): $\delta 11.4\left(\mathrm{CH}_{3}\right), 20.2$ $\left(\mathrm{CH}_{2}\right), 41.0\left(\mathrm{NCH}_{2}\right), 56.5\left(\mathrm{C}_{\text {spiro }}\right), 101.9\left(\mathrm{C}-\mathrm{NO}_{2}\right), 112.7(\mathrm{C}=\mathrm{C}-\mathrm{O})$, 125.7, 126.1, 128.1, 131.4, 131.8, 133.4, 134.5 (Ar), $142.8(\mathrm{O}-\mathrm{C}-\mathrm{N})$, $155.2(\mathrm{C}=\mathrm{C}-\mathrm{O}), 173.0$ (NCO), 176.5 (CO), 207.0 (CO).

\section{Conflicts of interest}

The authors declare no competing financial interest.

\section{Acknowledgements}

Financial support of this research from Imam Khomeini International University, Iran is gratefully acknowledged.

\section{References}

1 E. Lionta, G. Spyrou, D. K Vassilatis and Z. Cournia, Curr. Top. Med. Chem., 2014, 14, 1923-1938.

2 J. Kim, H. Kim and S. B. Park, J. Am. Chem. Soc., 2014, 136, 14629-14638.

3 V. P. R. Gajulapalli, K. Lokesh, M. Vishwanath and V. Kesavan, RSC Adv., 2016, 6, 12180-12184.

4 M. D. Aytemir and B. Özçelik, Eur. J. Med. Chem., 2010, 45, 4089-4095.

5 D. Us, E. Gürdal, B. Berk, S. Öktem, T. Kocagöz, B. Çağlayan and D. D. Erol, Turk. J. Chem., 2009, 33, 803-812.

6 N. A. Keiko, L. G. Stepanova, M. G. Voronkov, G. I. Potapova, N. O. Gudratov and E. M. Treshchalina, Pharm. Chem. J., 2002, 36, 407-409.

7 L. Bonsignore, G. Loy, D. Secci and A. Calignano, Eur. J. Med. Chem., 1993, 28, 517-520.

8 N. Lashgari and G. M. Ziarani, ARKIVOC, 2012, (i), 277-320.
9 B. M. Trost and M. K. Brennan, Synthesis, 2009, 2009, 30033025.

10 A. Velazquez-Campoy, M. J. Todd and E. Freire, Biochemistry, 2000, 39, 2201-2207.

11 T. Ponpandian and S. Muthusubramanian, Synth. Commun., 2014, 44, 868-874.

12 R. C. Cioc, E. Ruijter and R. V. Orru, Green Chem., 2014, 16, 2958-2975.

13 X. B. Chen, Z. C. Liu, L. F. Yang, S. J. Yan and J. Lin, ACS Sustainable Chem. Eng., 2014, 2, 1155-1163.

14 R. Ghahremanzadeh, T. Amanpour and A. Bazgir, J. Heterocycl. Chem., 2009, 46, 1266-1270.

15 K. Rad-Moghadam and L. Youseftabar-Miri, Tetrahedron, 2011, 67, 5693-5699.

16 Y. Zou, Y. Hu, H. Liu and D. Shi, ACS Comb. Sci., 2011, 14, 3843.

17 A. Bazgir, G. Hosseini and R. Ghahremanzadeh, ACS Comb. Sci., 2013, 15, 530-534.

18 G. Mohammadi Ziarani, N. Hosseini Mohtasham, N. Lashgari, A. Badiei, M. Amanlou and R. Bazl, J. Nanostruct., 2012, 2, 489-500.

19 T. He, Q. Q. Zeng, D. C. Yang, Y. H. He and Z. Guan, RSC Adv., 2015, 5, 37843-37852.

20 K. Niknam and P. Abolpour, Monatsh. Chem., 2015, 146, 683690.

21 C. Wang, Y. H. Jiang and C. G. Yan, Chin. Chem. Lett., 2015, 26, 889-893.

22 K. Niknam, A. Piran and Z. Karimi, J. Iran. Chem. Soc., 2016, 13, 859-871.

23 J. Xie, X. Y. Xing, F. Sha, Z. Y. Wu and X. Y. Wu, Org. Biomol. Chem., 2016, 14, 8346-8355.

24 P. Muthuraja, S. Prakash, G. Siva, S. Muthusubramanian and P. Manisankar, ChemistrySelect, 2017, 2, 10071-10075.

25 M. Zhang, Q. Y. Fu, G. Gao, H. Y. He, Y. Zhang, Y. S. Wu and Z. H. Zhang, ACS Sustainable Chem. Eng., 2017, 5, 6175-6182.

26 A. Hasaninejad, F. Mandegani, M. Beyrati, A. Maryamabadi and G. Mohebbi, ChemistrySelect, 2017, 2, 6784-6796.

27 T. Liu, Y. H. Lai, Y. Q. Yu and D. Z. Xu, New J. Chem., 2018, 42, 1046-1051.

28 F. A. Tameh, J. Safaei-Ghomi, M. Mahmoudi-Hashemi and H. Shahbazi-Alavi, RSC Adv., 2016, 6, 74802-74811.

29 L. Zhang, J. Dong, X. Xu and Q. Liu, Chem. Rev., 2016, 116, 287-322.

30 N. Poomathi, J. Kamalraja, S. Mayakrishnan, D. Muralidharan and P. T. Perumal, Synlett, 2014, 25, 708712.

31 E. Safari, A. Maryamabadi and A. Hasaninejad, RSC Adv., 2017, 7, 39502-39511.

32 M. Bayat, F. Safari, S. Nasri and F. S. Hosseini, Monatsh. Chem., 2019, 1-8.

33 S. Nasri, F. S. Hosseini and M. Bayat, Tetrahedron, 2018, 74, 4409-4417.

34 M. Bayat and S. Nasri, Tetrahedron Lett., 2017, 58, 3107-3111. 35 S. Ghadiri, M. Bayat and F. S. Hosseini, Monatsh. Chem., 2019, 1-6.

36 G. M. Ziarani, R. Moradi and N. Lashgari, ARKIVOC, 2016, (i), 1-81. 